Canadian

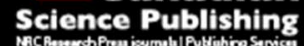

Canadian Journal of Chemistry Revue canadienne de chimie

\title{
Effect of alloy on the evolution of created magnons in the multilayer system $\mathrm{Fe}_{\mathrm{x}} \mathrm{Co}_{1-\mathrm{x}} / \mathrm{Cu}$
}

\begin{tabular}{|r|l|}
\hline Journal: & Canadian Journal of Chemistry \\
\hline Manuscript ID & cjc-2017-0247.R1 \\
\hline Manuscript Type: & Article \\
\hline Date Submitted by the Author: & $29-$ May-2017 \\
\hline Complete List of Authors: & $\begin{array}{l}\text { AIT LABYAD, Nadia; Universite Ibn Tofail Kenitra, Department of Physics } \\
\text { QACHAOU, Ahmed; Universite Ibn Tofail Kenitra Faculte des Sciences } \\
\text { FAHMI, Atika; Universite Ibn Tofail Kenitra Faculte des Sciences } \\
\text { FAHOUME, Mounir; Universite Ibn Tofail Kenitra Faculte des Sciences }\end{array}$ \\
\hline $\begin{array}{r}\text { Is the invited manuscript for } \\
\text { consideration in a Special } \\
\text { Issue?: }\end{array}$ & N/A \\
\hline Keyword: & $\begin{array}{l}\text { Magnon, Excitation spectra, Magnetization by spin, Gap of magnon } \\
\text { creation, Permitted band for magnon creation }\end{array}$ \\
\hline \multicolumn{2}{|c}{} \\
\hline
\end{tabular}

\section{SCHOLARONE ${ }^{m}$}

Manuscripts 


\title{
Effect of alloy on the evolution of created magnons in the multilayer system $\mathrm{Fe}_{x} \mathrm{Co}_{1-x} / \mathrm{Cu}$
}

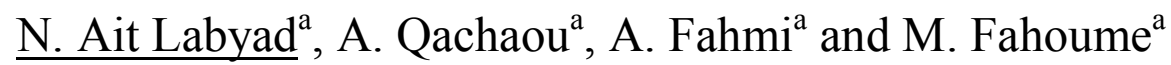

${ }^{\mathrm{a}}$ Department of physics, University Ibn Tofaïl, 14000 Kenitra-Morocco

Corresponding author: Dr. Nadia Ait Labyad

E-mail: n.aitlabyad012@gmail.com

Phone: +212650790065

Fax: +212537329433 


\begin{abstract}
The properties of magnons created in $\mathrm{FeCo} / \mathrm{Cu}$ multilayers are studied in the framework of the Heisenberg model. The excitation spectra, gap of creation, lifetimes and magnetization are calculated. The magnetization agrees well with measured values and it undergoes a 3D-2D transition when the temperature increases.
\end{abstract}

Keywords: Magnon, Excitation spectra, Magnetization by spin, Gap of magnon creation, Permitted band for magnon creation. 


\section{Introduction}

Magnetic multilayer systems are an important subject of basic and applied research. Several studies have attempted to understand the differences between their properties and those of bulk analogous systems [1, 2, and 3].

As the Ising model do not give satisfactory results for the ferromagnetism theory, an effort has been made to exploit the realistic model of Heisenberg by several authors [4, 5, 6, and 7], in order to calculate the spin-wave energies and other phenomena of magnetism at high temperatures and up to the Curie temperature. At lower temperatures, difficulties arise which were commented by Anderson [8], and treated with several modern approaches as Monte Carlo method [9], SLDFT [10], LSDA [11] and ab initio many-body perturbation theory, where magnon dispersion curves of the tetragonal FeCo compounds grown on $\mathrm{Pd}$, $\mathrm{Ir}$, and $\mathrm{Rh}$, respectively have been calculated [12]. A calculation analogous to ours, at a finite temperature is made by Blauch-Nauciel [7] which these magnetic excitations have been treated by a Selfconsistent theory of spin waves in interaction.

We adopt the Heisenberg model as we believe it to be the most appropriate method to describe the studied system since it realized for the case of localized moments, which $\mathrm{FeCo}$ alloy is suitable candidate, as it is characterized by a smaller proportion of roaming electrons than those localized. And the emergence of these new properties would be mainly caused by the symmetry breaking along the axis $\mathrm{Oz}$ perpendicular to the film. Under the effect of this break in symmetry, the filling of the sub-bands $d_{\uparrow}$ and $d_{\downarrow}$ of the transition metals can undergo, in the case of thin films, important modifications. We study the properties of magnons created in multilayered $\mathrm{Co}_{1-x} \mathrm{Fe}_{x} / \mathrm{Cu}$ alloys. The Hamiltonian of Heisenberg contains terms of exchange $(\mathrm{J})$, an applied magnetic field $\left(h=g \mu_{B} H\right)$ and magnetocrystalline anisotropy $\left(\Delta_{i j}\right)$. We calculated the excitation spectrum and the magnetization per site. 


\section{Spin Hamiltonian}

For $\mathrm{Co}_{1-x} \mathrm{Fe}_{x}$ layers made by $N$ ferromagnetic planes parallel to Oxy, the spin Hamiltonian is:

$$
\begin{aligned}
& \mathrm{H} \equiv-\sum_{<i j>} J_{i j}^{\|}\left(\Delta_{i j}\left(S_{i}^{x} S_{j}^{x}+S_{i}^{y} S_{j}^{y}\right)+S_{i}^{z} S_{j}^{z}\right)-\sum_{<i i^{\prime}>} J_{i i^{\prime}}^{\perp(\text { inout })}\left(S_{i}^{x} S_{i^{\prime}}^{x}+S_{i}^{y} S_{i^{\prime}}^{y}+S_{i}^{z} S_{i^{\prime}}^{z}\right) \\
& -g \mu_{B} H \sum_{i j} S_{i}^{z} S_{j}^{z}
\end{aligned}
$$

$\left(J^{\|}, J^{\perp i n}\right)$ and $J^{\perp \text { out }}$ are the exchange integrals between neighbors located in the same plane and in two different planes respectively. Then, by successive transformations of HolsteinPrimakof [13] and Fourier [14] in the xoy plane, we wrote $\mathrm{H}=$ in the case where only the effect of the exchange is taken into account:

$$
\mathrm{H}=\sum_{l_{z}, m}^{N} \sum_{k_{\|}} A_{l_{z} m}\left(k_{\|}, x\right) a_{k_{\|, l_{z}}^{+}}^{+} a_{k_{\|, m}}
$$

with: $\quad A_{l_{z} m}\left(k_{\|}, x\right)=\left\{4 x_{C o}^{2} J_{C o C o}^{\|} S_{C o}\left(2-\Delta_{C o C o} \sum\left(k_{\|}\right)\right)+4 x_{F e}^{2} J_{F e F e}^{\|} S_{F e}\left(2-\Delta_{F e F e} \sum\left(k_{\|}\right)\right)+\right.$ $8 x_{C o} x_{F e} J_{C o F e}^{\|}\left(S_{C o}+S_{F e}-\Delta_{C o F e} \sqrt{S_{C o} S_{F e}} \Sigma\left(k_{\|}\right)\right)+x_{C o}^{2} S_{C o}\left(8 J_{C o C o}^{\perp \text { in }}+J_{C o C o}^{\perp o u t}\right)+x_{F e}^{2} S_{F e}\left(8 J_{F e F e}^{\perp i n}+J_{F e F e}^{\perp o u t}\right)$ $\left.+x_{C o} x_{F e}\left(S_{C o}+S_{F e}\right)\left(16 J_{C o F e}^{\perp i n}+J_{C o F e}^{\perp o u t}\right)\right\} \delta_{l_{z}, m}-\left\{\left(8 x_{C o}^{2} S_{C o} J_{C o C o}^{\perp i n}+8 x_{F e}^{2} S_{F e} J_{F e F e}^{\perp i n}+16 x_{C o} x_{F e} \sqrt{S_{C o} S_{F e}} J_{C o F e}^{\perp i n}\right) \times\right.$ $\left.\Lambda\left(k_{\|}\right) \cos \left(k_{z} a / 2\right)+\frac{1}{2}\left(x_{C o}^{2} S_{C o} J_{C o C o}^{\perp \text { out }}+x_{F e}^{2} S_{F e} J_{F e F e}^{\perp \text { out }}+x_{C o} x_{F e} \sqrt{S_{C o} S_{F e}} J_{C o F e}^{\perp o u t}\right) \cos \left(k_{z} a\right)\right\}\left(\delta_{l_{z}, m+1}+\delta_{l_{z}, m-1}\right)$ $C o_{1-x} F e_{x} / C u$ is bcc structure [15]: $\sum\left(k_{\|}\right)=\cos \left(k_{x} a\right)+\cos \left(k_{y} a\right) ; \Lambda\left(k_{\|}\right)=\cos \left(k_{x} \frac{a}{2}\right) \cos \left(k_{y} \frac{a}{2}\right)$

\subsection{Excitation spectra $E_{l_{z}}\left(k_{\|}, x\right), x=x_{F e}$}

For a system made by c layers each containing p magnetic planes, the spectra of the created magnons are obtained by following the same procedure that we have previously established in [16] for solving: $\operatorname{det} M\left(k_{\|}, x\right)=\left|A\left(k_{\|}, x\right)-E\left(k_{\|}, x\right) I\right|=0$. The dynamic matrix $M\left(k_{\|}, x\right)$ is built as $M\left(k_{\|}, x\right)=C\left(k_{\|}, x\right) \otimes P\left(k_{\|}, x\right)$ where $C\left(k_{\|}, x\right)$ and $P\left(k_{\|}, x\right)$ are matrices of 
orders c and p respectively with $N=c x p$. To diagonalize $M\left(k_{\|}, x\right)$, we first diagonalize $P\left(k_{\|}, x\right)$ giving $\mathrm{p}$ values $E_{i}\left(k_{\|}, x\right)$ for a given layer. Then, we diagonalize $C\left(k_{\|}, x\right)$ for c layers leading to $c \times p$ different energies $E_{i}^{j}\left(k_{\|}, x\right)$ with $(1 \leq i \leq p)$ and $(1 \leq j \leq c)$. For example, (Figure. 1$)$ gives the spectra for different $x$ and for a system with $p=3$ and $c=5$.

Otherwise, assuming that each magnetic plane $l_{z}$ only interacts with its two adjacent planes $l_{z} \pm 1$, we have $J^{\perp \text { out }}=0$ and $J^{\perp \text { in }}=J^{\perp}$. The spectrum is then given by: $E_{l_{z}}\left(k_{\|}, x\right)=\alpha\left(k_{\|}, x\right)-\beta\left(k_{\|}, x\right) \cos \left(\frac{k_{z} a}{2}\right)$, where :

$\alpha\left(k_{\|}, x\right)=8 x_{C o}^{2} S_{C o}\left(J_{C o C o}^{\|}+J_{C o C o}^{\perp}\right)+8 x_{F e}^{2} S_{F e}\left(J_{F e F e}^{\|}+J_{F e F e}^{\perp}\right)+8 x_{C o} x_{F e}\left(S_{C o}+S_{F e}\right)\left(J_{C o F e}^{\|}+2 J_{C o F e}^{\perp}\right)$ $-4\left[x_{\mathrm{Co}}^{2} S_{\mathrm{Co}} J_{\mathrm{CoCo}}^{\|} \Delta_{\mathrm{CoCo}}+x_{\mathrm{Fe}}^{2} S_{\mathrm{Fe}} J_{\mathrm{FeFe}}^{\|} \Delta_{\mathrm{FeFe}}+2 x_{\mathrm{Co}} x_{\mathrm{Fe}} J_{\mathrm{CoFe}}^{\|} \Delta_{\mathrm{CoFe}} \sqrt{S_{\mathrm{Co}} S_{F e}}\right] \sum\left(k_{\|}\right)=e_{1}(x)-e_{2}\left(k_{\|}, x\right)$ and $\beta\left(k_{\|}, x\right)=8\left(x_{C o}^{2} S_{C o} J_{C o C o}^{\perp}+x_{F e}^{2} S_{F e} J_{F e F e}^{\perp}+2 x_{C o} x_{F e} \sqrt{S_{C o} S_{F e}} J_{C o F e}^{\perp}\right) \Lambda\left(k_{\|}\right)$.The function $E_{l_{z}}\left(k_{\|}, x\right)$ is bounded in $E_{m} \leq E_{l_{z}}\left(k_{\|}, x\right) \leq E_{M}$. The lower and upper bounds given by: $E_{m}=E_{l_{z}}\left(k_{\|}, k_{z}=0, x\right)=\alpha-\beta$ and $E_{M}=E_{l_{z}}\left(k_{\|}, k_{z}=\frac{2 \pi}{a}, x\right)=\alpha+\beta$ defining a width of "permitted band" of creation of the magnons by: $W=\left|E_{M}-E_{m}\right|=16\left|\left[x^{2}\left(S_{C o} J_{C o C o}^{\perp}+S_{F e} J_{F e F e}^{\perp}-2 \sqrt{S_{C o} S_{F e}} J_{C o F e}^{\perp}\right)-2 x\left(S_{C o} J_{C o C o}^{\perp}-\sqrt{S_{C o} S_{F e}} J_{C o F e}^{\perp}\right)+S_{C o} J_{C o C o}^{\perp}\right] \Lambda\left(k_{\|}\right)\right|$

A gap of energy controlling this creation is also defined at the point $\Gamma$ of the BZ by:

$$
\begin{gathered}
E_{g}(x)=E_{m}(0, x) \text {. So, } E_{g}(x)=x_{C o}^{2} S_{C o} J_{C o C o}^{\|}\left(1-\Delta_{C o C o}\right)+x_{F e}^{2} S_{F e} J_{F e F e}^{\|}\left(1-\Delta_{F e F e}\right)+x_{C o} x_{F e} \times \\
{\left[J_{C o F e}^{\|}\left(S_{C o}+S_{F e}-2 \Delta_{C o F e} \sqrt{S_{C o} S_{F e}}\right)+2 J_{C o F e}^{\perp}\left(S_{C o}+S_{F e}-\sqrt{S_{C o} S_{F e}}\right)\right]}
\end{gathered}
$$

A lifetime of these created magnons can thus be deduced such that:

$\frac{1}{\tau(x)}=\frac{2 \pi}{\hbar}\left|E_{g}(0, x)\right|^{2} D\left(x, E_{F}\right)$. The density of states of the alloy can be expressed in a rigid 
band approximation by: $D\left(x, E_{F}\right)=(1-x) D_{C o}\left(E_{F}\right)+x D_{F e}\left(E_{F}\right)$ with $D_{C o}\left(E_{F}\right)=3.54\left(\mathrm{eV}^{-1} / \mathrm{at}\right)$

[17] and $D_{F e}\left(E_{F}\right)=4.16\left(\mathrm{eV}^{-1} / \mathrm{at}\right)[18]$.

Thus, (i) by increasing $\mathrm{x}$, the creation of the magnon is favored as reflected by increasing $W\left(k_{\|}, x\right)$ (Figure.2), but with a lowered stability (Figure.3) for a fixed exchange ratio $J_{\mathrm{CoFe}}^{\perp} / J_{\mathrm{CoFe}}^{\|}$. These excitations created disappear even rapidly (Figure.4). (ii) Whereas for a fixed $x$, it is the reinforcement of the exchange perpendicular to the plane of the layers which reinforces the stability (Figure.3).

\subsection{Magnetization by spin}

The magnetization by spin is expressed in the Brillouin zone (BZ) and for a surface $\mathrm{s}$ of the unit cell by:

$M_{c a l}^{z}(T, x)=1-\frac{1}{S} \frac{1}{N} \frac{S}{(2 \pi)^{2}} \sum_{l_{z}=1}^{N} \int_{B Z}<a_{k_{\|}, l_{z}}^{+} a_{k_{\|}, l_{z}}>d k_{x} d k_{y}=1-\frac{1}{S} \frac{1}{N} \frac{S}{(2 \pi)^{2}} \sum_{l_{z}=1}^{N} \int_{B Z} \frac{1}{\exp \left(\frac{E_{l_{z}}\left(k_{\|}, x\right)}{k_{B} T}\right)-1} d k_{x} d k_{y}$ $E_{l_{z}}\left(k_{\|}, x\right)=e_{1}(x)-e_{2}(0, x) \sum\left(k_{\|}\right)-\beta(0, x) \Lambda\left(k_{\|}\right) \cos \frac{k_{z} a}{2}$. Then, using the same calculation as in

[19], we have for $k_{B} T<<E(k, x)<\frac{k_{B} T_{C}}{3}$ :

$$
M_{c a l}^{z}(T, x)=g_{3 / 2}(z) \frac{1}{(2 \pi)^{3 / 2}} \frac{1}{\left[e_{2}(0, x)+\beta(0, x)\right][\beta(0, x)]^{\frac{1}{2}}}\left(k_{B} T\right)^{3 / 2} .
$$

$g_{3 / 2}(z)$ is the function of Bose. $M^{z}(T, x)$ behaves in $T^{3 / 2}$ Bloch-law as usual for 3D systems.

While, for higher temperatures such as $E(k, x)<<k_{B} T<\frac{k_{B} T_{C}}{3}$, it behaves in $T \ln T$ as in 2D systems:

$$
M_{c a l}^{z}(T, x)=\frac{1}{2 \pi S} \frac{k_{B} T}{\left[e_{2}(0, x)+\beta(0, x)\right]} \ln \frac{2 k_{B} T}{\left(e_{1}(x)-2 e_{2}(0, x)\right)\left(1+\sqrt{1-\left(\frac{\beta(0, x)}{e_{1}(x)-2 e_{2}(0, x)}\right)^{2}}\right)}
$$


The agreement of $M_{c a l}^{z}(T, x)$ with the results of measurements $M_{\exp }^{z}(T, x)$ is, as shown in (Figure.5), very satisfactory. The values deduced for the integrals of exchange obtained (Table.1) are in good agreement with what is usually given for this type of samples based on $3 d$ transition metals [16, 19-22].

\section{Conclusion}

The spectrum $E\left(k_{\|}, x\right)$ of magnetic excitations created in the magnetic multilayers $\mathrm{Co}_{1-x} \mathrm{Fe}_{x} / \mathrm{Cu}$ is calculated. A "permitted band" of creation is determined. It is characterized by a width $\mathrm{W}$, a creation gap and a lifetime of these magnons. When the iron content $x$ increases, the creation is favored. However, the stability and lifetime of these created excitations are disadvantaged.

The $M_{c a l}^{z}(T, x)$ curves show a transition 3D-2D when $\mathrm{T}$ increases. The agreement with $M_{\text {exp }}^{z}(T, x)$ is good giving values of the integrals of exchange usually found for similar multilayers. 


\section{References}

(1) Ercole, A.; Lew, W. S.; Lauhoff, G.; Kernohan, E. T. M.; Lee, J.; Bland, J. A. C. Phys. Rev. 2000, 62, 6429

(2) Yoshire, A.; Suzuki, Y. and Katayama, T. JPSJ. 1996, 65, 1469

(3) Kerkmann, D.; Wolf, J.A.; Pescia, D.; Woike, D.; Grünberg, P. Solid State Commun. $1989,72,963$

(4) Weiss, P. R. Phys. Rev. 1948 74, 27

(5) Yvon, J. Cahiers de Physique. 1945. 1948; J. Physique Rad. 1947, 8, 182; J. Physique. Rad. 1949, 10, 373

(6) Mills, D. L.; Maradudin, A. A. J. Phys. Chem. Solids, Pergamon Press. 1967, 28, 1855

(7) Bloch-Nauciel, M. Journal of Physic. 1968, 29 (7), 694-704

(8) Anderson, P. W. Phys. Rev, 1950, 80, 922

(9) Takahashi, M. Phys. Rev. Lett. 1989, 62, 2313

(10) Pulikkotil, J.J.; Ke, L.; Van Schilfgaarde, M.; Kotani' T.; Antropov, P. V. Superconductor Science and Technology. 2010, 23, 5

(11) Katsnelson, M. I.; Lichtenstein, A. I. J. Phys. Condens. Matter. 2004, 16, 7439-7446

(12) Sasiolu, E.; Friedrich, C.; Blügel, S. Phys. Rev, B. 2013, 87, 020410(R)

(13) Holstein, T.; Primakoff, H. Phys. Rev. 1940, 58, 1908

(14) Politi, P.; Pini, M. G.; Rettori, A. Phys. Rev. B. 1992, 46, 8312

(15) Graff, I.L.; Traverse, A.; Geshev, j.; Teixeira, S. R.; Amaral, L. NIMB. 2007, 257, 424-427

(16) Kübler, J.; Eyert, V. Materials Science and Technology. 2007, 3A, 81-82

(17) Gautier, F. Dunod. 1973, 264

(18) Rupp, G.; Schester, E. JMMM. 1993, 121, 416-420

(19) Fahmi, A.; Qachaou, A. Physica B. 2009, 404, 2474-2480

(20) Elmoussaoui, H.; Fahmi, A.; Qachaou, A.; Fahoume, M. Physica E. 2014, 57, 149-154

(21) Mehdioui, M. ; Fahmi, A.; Qachaou, A.; Fahoume, M.; Lassri, H. JMMM. 2014, 352, 107

(22) Fahmi, A.; Elharfaoui, M.; Qachaou, A. JMMM. 2006, 303, 26-33; Fahmi, A. 2006 Thesis, Kenitra, Morocco 


\section{Figure captions:}

Figure.1: Excitation spectra $E_{N=15}\left(k_{\|}, x\right)$ calculated for $x$ variant and:

$$
J_{\mathrm{CoCo}}^{\|}=J_{\mathrm{CoFe}}^{\|}=J_{\mathrm{FeFe}}^{\|}=0.1, J_{\mathrm{CoCo}}^{\perp \text { in }}=J_{\mathrm{CoFe}}^{\perp \text { in }}=J_{\mathrm{FeFe}}^{\perp \text { in }}=1, J_{\mathrm{CoCo}}^{\perp \text { out }}=J_{\mathrm{CoFe}}^{\perp \text { out }}=J_{\mathrm{FeFe}}^{\perp \text { out }}=0.5
$$

Figure.2: Band width $W\left(k_{\|}, x\right)$ of created magnon for variant $x$ and fixed $k_{\|}$

Figure.3: The phase diagram $\Delta\left(J^{\perp} / J^{\|}\right)$for varying $x$ and for $E_{g}=0$

Figure.4: The lifetime of magnons: $\tau(x)$

Figure.5: Comparison with experimental results

$$
\begin{aligned}
& M_{\text {cal }}^{z}(T, x): \mathrm{x}=0-; 0.7-\longrightarrow ; 1---- \\
& M_{\mathrm{exp}}^{z}(T, x): \mathrm{x}=0(*) ; 0.7(\circ) ; 1(\bullet)[12] .
\end{aligned}
$$




\section{$\underline{\text { Tables : }}$}

Table.1: Values of the exchange and the magnetocrystalline anisotropy

\begin{tabular}{ccccccccc}
\hline$J_{\mathrm{CoCo}}^{\|}$ & $J_{\mathrm{FeFe} e}^{\|}$ & $J_{\mathrm{CoFe}}^{\|}$ & $J_{\mathrm{CoCo}}^{\perp}$ & $J_{\mathrm{FeFe}}^{\perp}$ & $J_{\mathrm{CoFe}}^{\perp}$ & $\Delta_{\mathrm{CoCo}}$ & $\Delta_{\mathrm{FeFe}}$ & $\Delta_{\mathrm{CoFe}}$ \\
\hline 8 & 7 & 8 & 100 & 98 & 105 & 1 & 0.4 & 0.7 \\
8 & 9 & 9.5 & 90 & 83 & 95 & 0.9 & 0.3 & 0.6 \\
7.9 & 8.8 & 9.7 & 79 & 88 & 97 & 0.8 & 0.4 & 0.5 \\
\hline
\end{tabular}




\section{Figures:}
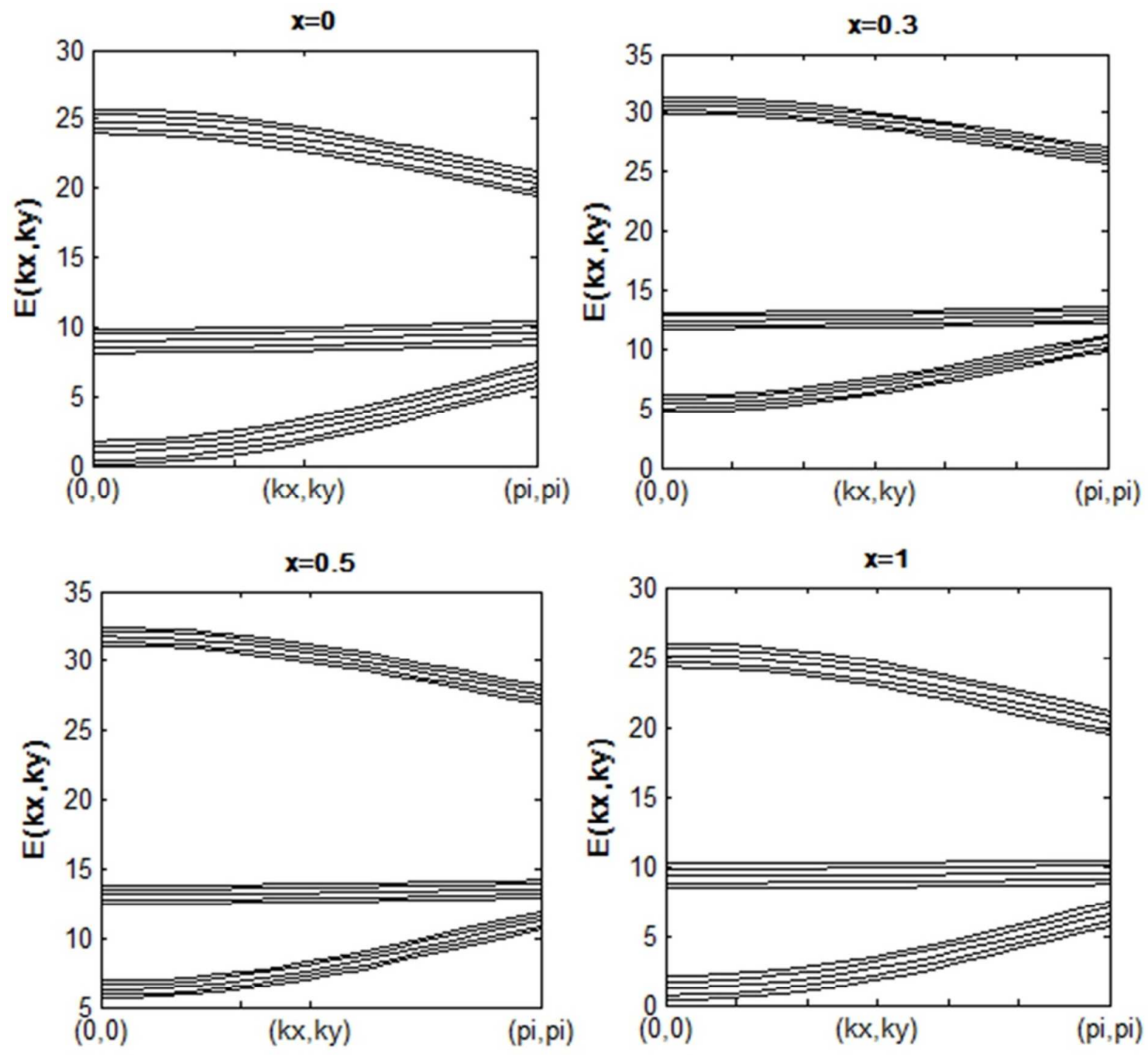

Figure.1: Excitation spectra $E_{N=15}(k, x)$ calculated for variant $x$ and

$$
J_{\mathrm{CoCo}}^{\|}=J_{\mathrm{CoFe}}^{\|}=J_{\mathrm{FeFe}}^{\|}=0.1, J_{\mathrm{CoCo}}^{\perp \text { in }}=J_{\mathrm{CoFe}}^{\perp \text { in }}=J_{\mathrm{FeFe}}^{\perp \text { in }}=1, J_{\mathrm{CoCo}}^{\perp \text { out }}=J_{\mathrm{CoFe}}^{\perp \text { out }}=J_{\mathrm{FeFe}}^{\perp \text { out }}=0.5
$$




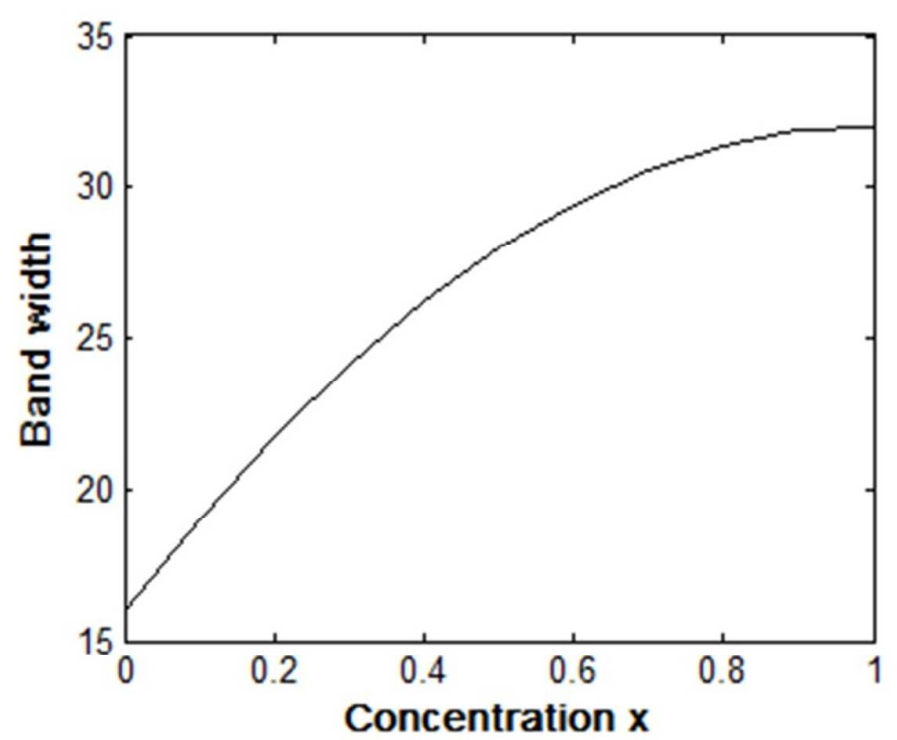

Figure.2: Band width $W\left(k_{\|}, x\right)$ of created magnon for variant $x$ and fixed $k_{\|}$ 


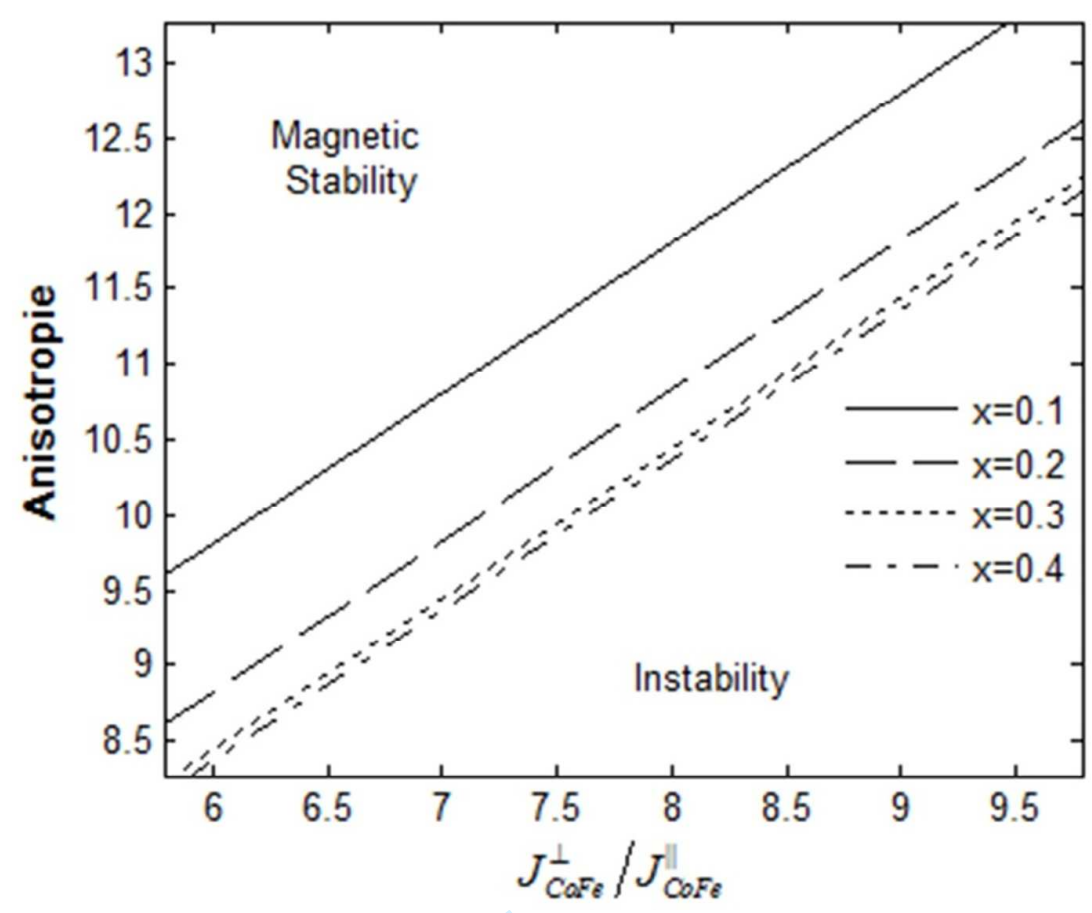

Figure.3: The phase diagram $\Delta\left(J^{\perp} / J^{\|}\right)$for varying $x$ and for $E_{g}=0$ 


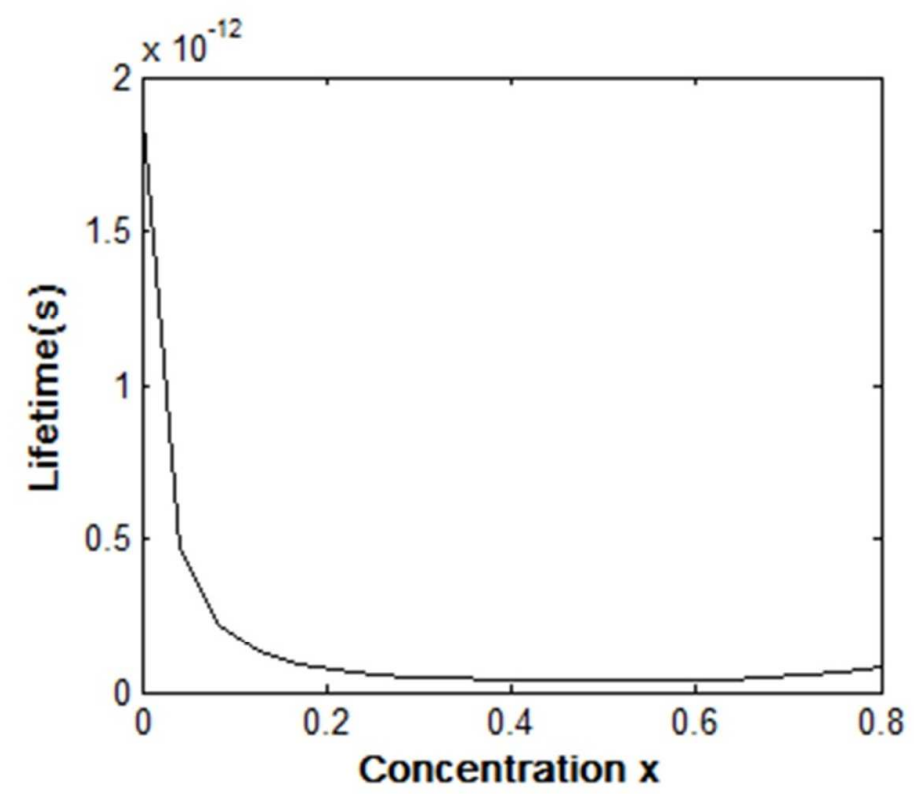

Figure.4: The lifetime of magnons: $\tau(x)$ 


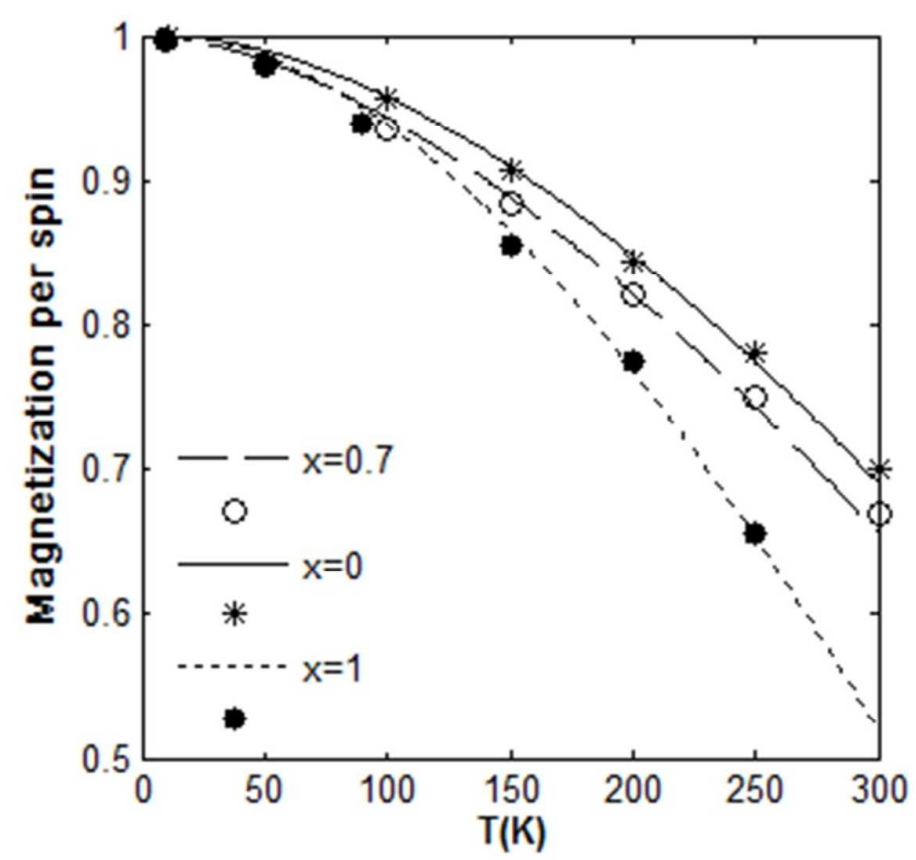

Figure.5: Comparison with experimental results

$$
\begin{aligned}
& M_{c a l}^{z}(T, x): \mathrm{x}=0 \longrightarrow ; 0.7 \longrightarrow 1---- \\
& M_{\exp }^{z}(T, x): \mathrm{x}=0(*) ; 0.7(\circ) ; 1(\bullet)[21] .
\end{aligned}
$$

